\title{
The genus Eodendrus Belokobylskij (Hymenoptera, Braconidae, Doryctinae) from China, with description of a new species
}

\author{
Man-man Wang ${ }^{\dagger}$, Jiang-li Tan ${ }^{\ddagger}$, Jun-hua He`, Xue-xin Chen ${ }^{\prime}$ \\ State Key Laboratory of Rice Biology, Institute of Insect Science, College of Agriculture and Biotechnology, \\ Zhejiang University, Hangzhou 310029, China \\ † urn:lsid:zoobank.org:author:CEF5FDB0-A37A-4E7B-9181-BF9CF47D763E \\ † urn:lsid:zoobank.org:author:71A3B5FF-D605-4284-ACD9-94AF5FF134B0 \\ § urn:lsid:zoobank.org:author:CDFF38D9-E9AE-4C8F-99CE-23151D3878F3 \\ | urn:lsid:zoobank.org:author:0054AC5F-7F6D-4811-A560-90983889C861 \\ Corresponding author: Xue-xin Chen (xxchen@zju.edu.cn)
}

Academic editor: Kees van Achterberg| Received 18 August 2009 | Accepted 8 September 2009 | Published 9 November 2009

urn:lsid:zoobank.org:pub:22803684-2D73-4751-9A5F-4A98998FA3D3

Citation: Wang M-M, Tan J-L, He, J-H, Chen X-X (2009) The genus Eodendrus Belokobylskij (Hymenoptera, Braconidae, Doryctinae) from China, with description of a new species. ZooKeys 27: 43-50. doi: 10.3897/zookeys.27.252

\begin{abstract}
The genus Eodendrus Belokobylskij (Hymenoptera: Braconidae, Doryctinae) from China is revised. Three species are recognized, of which a new species, E. reticulatus Wang \& Chen, sp. n., is described and illustrated, and Eodendrus hoabinicus Belokobylskij \& Long, 2005 recorded in China for the first time. A key to the species of this genus is updated to include the new species. The type specimens are kept in the Parasitic Hymenoptera Collection, Zhejiang University, Hangzhou (ZJUH).
\end{abstract}

\section{Keywords}

Hymenoptera, Braconidae, Doryctinae, Dendrosotinus, Eodendrus, new species, new record, Oriental region, China

Copyright $M-M$. Wang et al. This is an open access article distributed under the terms of the Creative Commons Attribution License, which permits unrestricted use, distribution, and reproduction in any medium, provided the original author and source are credited. 


\section{Introduction}

Eodendrus Belokobylskij was set up as an subgenus of the genus Dendrosotinus Telenga (Hymenoptera, Braconidae, Doryctinae) based on the Eastern Palaearctic species D. eous Belokobylskij, 1988 (Belokobylskij 1988). Later, Eodendrus Belokobylskij was raised to generic status (Belokobylskij et al. 2005). To date eight species of the genus Eodendrus Belokobylskij have been known in the world, of which one occurs in the Palaearctic, five in the Oriental, and two in the Afrotropical regions (Granger 1949, Belokobylskij 1988, Belokobylskij et al. 2005). Only one species of the genus - Eodendrus petiolatus has been recorded in China, which occurs in Guangxi province. During our study of Chinese materials of the genus Eodendrus, we discovered a new species and a species that have hitherto not been recorded from China. In the present paper, the new species is described and illustrated, the new record listed, and the key to the genus Eodendrus, as published by Belokobylskij et al. (2005), modified.

\section{Material and methods}

The terminology and measurements used follow van Achterberg $(1979,1988)$. Additional sources for the description of sculpture and setation are Belokobylskij et al. (2005). All descriptions and measurements were made under a Leica MZ $12.5 \mathrm{mi}-$ croscope, and figures made under ZEISS Stemi SV6 microscope. Type specimens and other materials are deposited in the Parasitic Hymenoptera Collection of the Zhejiang University, Hangzhou, China (ZJUH).

\section{Key to the species of the genus Eodendrus Belokobylskij}

(modified from Belokobylskij et al. 2005)

1. First subdiscal cell widened medially and closed apically almost at level of $\mathrm{m}$-cu vein; hind coxa with small basoventral tubercle; first tergite high (in lateral view); larger, body length $7.0 \mathrm{~mm}$. South Africa

E. africanus Belokobylskij, 2005

- $\quad$ First subdiscal cell not or weakly widened medially and usually closed apically distinctly before level of m-cu vein (except E. conspicuus); hind coxa without basoventral tubercle; first tergite usually not high (lateral view); smaller, body

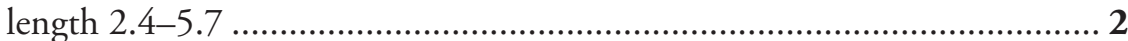

2. Length of mesosoma 2.4-2.5 times its height; mesoscutum weakly and roundly raised above pronotum; second and third metasomal tergites widely yellow laterally and brown to reddish brown medially; body length 3.9-4.2 mm. (Male) Vietnam ................ E. elongatus Belokobylskij \& Long, 2005

- $\quad$ Length of mesosoma 2.0-2.2 times its height; mesoscutum highly and almost perpendicularly raised above pronotum 
3. First tergite with small spiracular tubercles; acrosternite of female 0.350.4 times as long as first tergite; length of first tergite 1.4-1.6 times its apical width; lateral depressions of second tergite at most weakly convergent

- $\quad$ First tergite with long spiracular tubercles which are directed somewhat downwards; acrosternite of female 0.45-0.6 times as long as first tergite; length of first tergite 1.6-2.3 times its apical width; lateral depressions of second tergite distinctly convergent.

5

4. 3-SR vein of fore wing 0.5-0.6 times SR1, first subdiscal cell apically closed distinctly before m-cu vein; length of second tergite 1.1-1.15 times its basal width; body dark reddish brown; antenna 28-31-segmented; smaller, body length 3.0-3.4 mm. Russia (south of Far East), Japan, Korea

E. eous (Belokobylskij, 1988)

- $\quad 3-S R$ vein of fore wing 0.7 times SR1, first subdiscal cell apically closed almost at level of m-cu vein; length of second tergite 0.8-0.9 times its basal width; body usually light brown with dark spots; antennae 39-segmented; larger, body length 4.1-5.7 mm. Madagascar.

E. conspicuus (Granger, 1949)

5. Length of second tergite 1.6 times its basal width; length of first tergite 2.3 times its apical width; eyes sparsely, shortly setose. body length $4.2 \mathrm{~mm}$. China (Guangxi)

E. petiolatus Belokobylskij \& Chen, 2005

- $\quad$ Length of second tergite 0.9-1.3 times its basal width; length of first tergite 1.6-2.1 times its apical width; eyes glabrous 6

6. Transverse diameter of eye 1.4 times as long as temple (dorsal view); second submarginal cell of fore wing 3.4 times as long as its maximum width; vertex almost smooth medially; third tergite almost entirely smooth, very finely coriaceous laterally; scapus 1.25 times as long as its maximum width; body length $2.4 \mathrm{~mm}$. Vietnam....

E. flavus Belokobylskij \& Long, 2005

- $\quad$ Transverse diameter of eye twice as long as temple (dorsal view); second submarginal cell of fore wing 2.6-3.0 times as long as its maximum width; vertex distinctly and densely granulate, partly with dense and fine transverse striation; third tergite always sculpture basally, smooth medioapically.... 7

7. Lateral furrows of second tergite deep, distinctly carinate along interior margin, strongly posteriorly convergent; basal width of middle area 3.1 times its apical width; POL 2.0 times Od and 0.86 times OOL; dorsal hairs of hind tibia long, 0.8-1.36 times as long as apical width of tibia; scutellum finely granulate-coriaceous; body length $3.2 \mathrm{~mm}$. Vietnam

E. convergens Belokobylskij, 2005

- Lateral furrows of second tergite shallow, not carinate along interior margin, less distinctly posteriorly convergent; basal width of middle area 1.5-2.5 times its apical width; POL 1.4-1.6 times Od and 0.5-0.6 times OOL; dorsal hairs of hind tibia short, $0.5-0.8$ times as long as apical width of tibia 
8. Length of first tergite 2.1 times its apical width, maximum width of first tergite 2.0 times minimum width; spiracular tubercles of first tergite distinct and long, about 0.4 times basal width of first tergite; second tergite irregularly reticulate; third tergite reticulate in mediobasal 0.55 and the color of this area darker than the rest of third tergite. China (Hainan) .......E. reticulatus sp. $\mathbf{n}$.

- $\quad$ Length of first tergite 1.6-1.7 times its apical width, maximum width of first tergite 2.3 times minimum width; spiracular tubercles of first tergite relatively shorter; second tergite densely striate, with dense reticulation between striae; third tergite finely striate in mediobasal 0.25 and with the same color as the rest of third tergite. Vietnam, China....

E. hoabinicus Belokobylskij \& Long, 2005

\section{Eodendrus petiolatus Belokobylskij \& Chen, 2005}

Eodendrus petiolatus Belokobylskij et al., 2005: 2740.

Type material. Holotype female (ZJUH): China, Guangxi, Tianlin, $24^{\circ} 17^{\prime} 38^{\prime \prime} \mathrm{N}$, 106¹3'44"E, 278m elev., 31.V.1982, He Jun-hua, No.822073.

Distribution. China (Guangxi).

\section{Eodendrus hoabinicus Belokobylskij \& Long, 2005}

Eodendrus hoabinicus Belokobylskij et al., 2005: 2737.

Examined material. Female (ZJUH): China, Hainan, Jianfengling, $18^{\circ} 42^{\prime} 25^{\prime \prime N}$, 10849'47"E, 1200m elev., 7.VI.2007, Liu Jing-xian, No.200702549.

Distribution. China (Hainan), Vietnam.

Notes. This species is new to the fauna of China. Type specimens are preserved in the Institute of Ecology and Biological Resources, Hanoi, Vietnam (IEBR).

\section{Eodendrus reticulatus Wang \& Chen, sp. n.} urn:lsid:zoobank.org:act:44F720A1-E76D-43B6-BCD1-2F5395CECBE3

Figs $1-11$

Type material. Holotype female (ZJUH): China, Hainan, Jianfengling, $18^{\circ} 42^{\prime} 25^{\prime \prime} \mathrm{N}$, 10849'47"E, 1200m elev., 7.VI.2007, Liu Jing-xian, No.200702509.

Female. Length of body $3.0 \mathrm{~mm}$, of fore wing $2.7 \mathrm{~mm}$.

Head (Figs 1, 2). Antenna broken, remaining antennal segments 27, scapus as long as its maximum width, third segment as long as fourth segment (Fig. 3), length of third and fourth segments 6.0 and 5.0 times their width; length of maxillary palp 


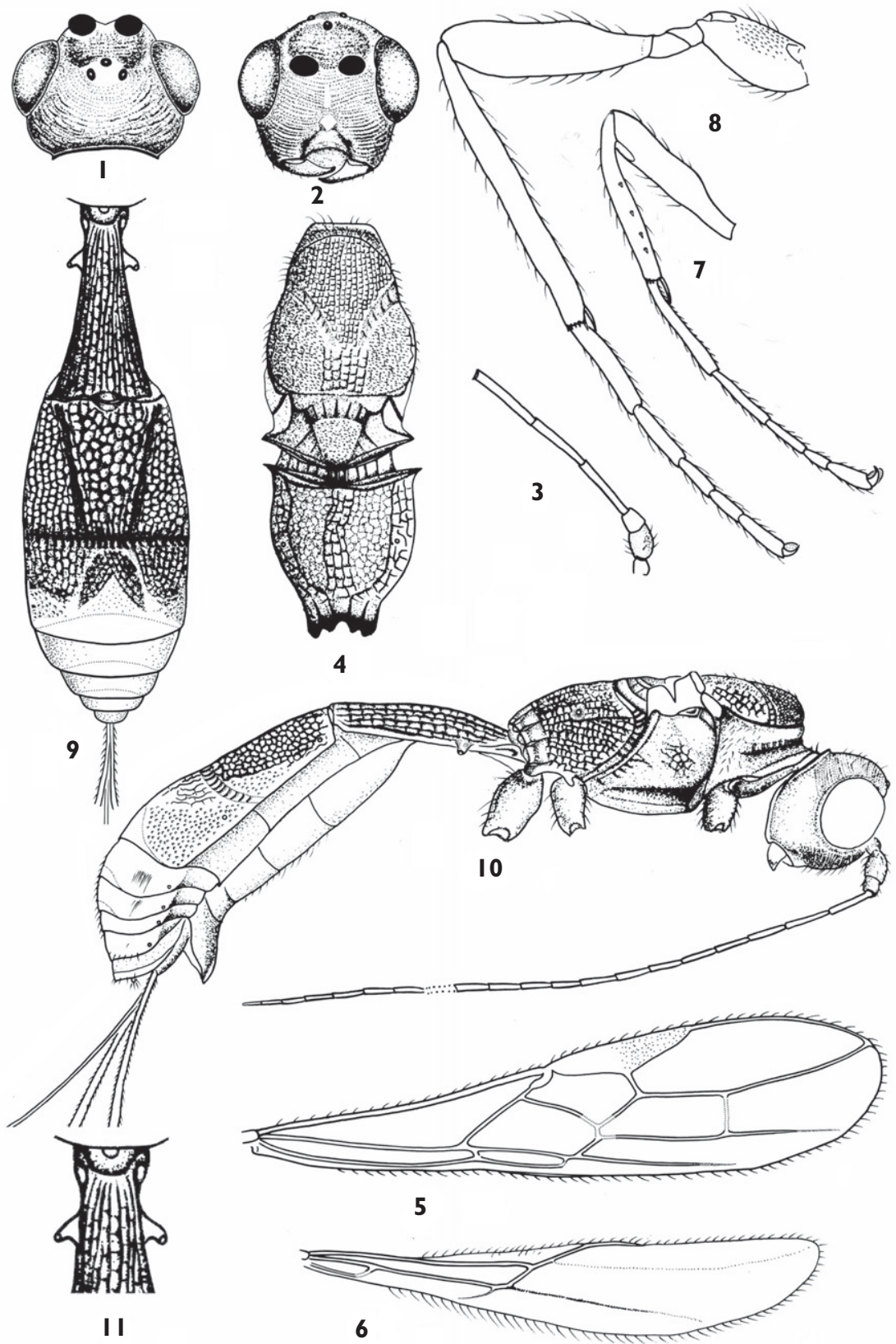

Figures I-I I. Eodendrus reticulatus Wang \& Chen, sp. n. $q \mathbf{I}$ head, dorsal aspect $\mathbf{2}$ head, frontal aspect $\mathbf{3}$ basal five segments of antenna $\mathbf{4}$ mesosoma, dorsal aspect $\mathbf{5}$ fore wing $\mathbf{6}$ hind wing $\mathbf{7}$ fore leg $\mathbf{8}$ hind leg 9 metasoma, dorsal aspect $\mathbf{I} \mathbf{0}$ habitus, lateral aspect $\mathbf{I} \mathbf{I}$ dorsope and spiracular tubercles of first tergite. 
1.7 times height of head; OOL: OD: $\mathrm{POL}=8: 3: 4$, posterior side of stemmaticum longer than lateral sides; eye glabrous; length of eye twice temple in dorsal view; head at level of eyes a little wider than at level of temple in dorsal view and temples behind eyes roundly narrowed; face distinctly transversely striate, densely clothed with long hairs; frons with rather irregular transverse striae and rugae; vertex finely transversely striate and granulate, clothed with short hairs; posterior part of temple finely striate, anterior part of temple almost smooth; length of malar space 1.6 times basal width of mandible and 0.56 times transverse diameter of eye in lateral view; longer diameter of eye 2.0 times length of temple.

Mesosoma (Figs 4, 10). Length of mesosoma 2.0 times its height; pronotal keel indistinct; side of pronotum with obsolescent short crenulation; mesoscutum highly and almost perpendicularly raised above pronotum, completely and densely clothed with short pubescence; medio-posteriorly with much irregular rugosity and the median carinae indistinct; notauli complete, deep in anterior half and shallow in posterior half, finely crenulate; mesopleuron densely granulate with rugae, coriaceous below precoxal sulcus; precoxal sulcus shallow, narrow and finely crenulate; scutellar sulcus shallow, with 4 carinae; scutellum slightly convex and coriaceous; propodeum without areola, with irregular rugae and reticulation; metapleuron with irregular rugosity.

Wings (Figs 5, 6). Fore wing 4.1 times as long as wide; pterostigma 4.2 times as long as wide; $r$ issued submedially from pterostigma; $r$ : 2-SR: SR1: 3-SR: $r-m=5: 10$ : 25: 14: 8; cu-a short and postfurcal; 1 -CU1: $2-\mathrm{CU} 1=2: 17$; 3 -CU1 antefurcal; $\mathrm{m}-\mathrm{cu}$ postfurcal, shorter than 2-SR; Second submarginal cell 2.6 times as long as its maximum width. Hind wing 5 times as long as wide; $\mathrm{M}+\mathrm{CU}: 1-\mathrm{M}=5: 11 ; \mathrm{m}$-cu present and stituated close to $1 \mathrm{r}-\mathrm{m}$.

Legs (Figs 7, 8). Fore tibia with 4 spines, fore tarsus 1.7 times as long as fore tibia; fore femur with a blister at basal 1/3 (Fig. 7); hind coxa without a baso-ventral tubercle (Fig. 8), its length 1.7 times maximum width, length of femur, tibia and basitarsus of hind leg 3.7, 8.8 and 6.0 times their width, respectively; hind tibia with short and semi-erect setae dorsally, setae about 0.6 times maximum width of tibia; hind tibia with 5 apical spines laterally; hind tarsus as long as hind tibia, basitarsus 0.5 times combined length of 2 nd-5th segments and 1.5 times second tarsus, third tarsal segment 0.8 times as long as telotarsus.

Metasoma (Figs 9, 10). First tergite gradually widened from base to apex, its maximum width 2.0 times its minimum width, its length 2.1 times its apical width and 1.5 times length of propodeum, its surface striate and coarsely granulate, with distinct and long spiracular tubercles at basal 0.24 and directed somewhat downwards, length of spiracular tubercle about 0.4 times basal width of first tergite; dorsope distinct, medium-sized (Fig. 11); acrosternite 0.45 times as long as first tergite; length of T2+3 1.7 times its width (Fig. 9), with a distinct sharp lateral margin; length of second tergite 0.9 times its width, with rather distinct, shallow, almost straight, posteriorly convergent longitudinal furrows; basal width of median area about 2.5 times its apical width (Fig. 9); second metasomal suture distinct, wide, curved and granulate; surface of $\mathrm{T} 2+3$ irregularly reticulate with the apical semicircular part smooth; remaining 
tergites smooth; ovipositor sheath 1.1 times as long as body, 1.2 times as long as fore wing, 3.7 times as long as hind tibia and 1.9 times as long as metasoma.

Colour. Brown; head and basal segments of antenna yellowish brown, apical segments dark brown; palpi pale yellowish; mesoscutum, scutellum and propodeum dark brown; fore coxa and tibia pale brown, tarsus yellowish brown but telotarsus dark brown; middle leg pale brown; hind leg yellowish brown but telotarsus dark brown; wing membrane brownish translucent, basal half of pterostigma pale and apical half dark brown; first tergite of metasoma dark brown, second tergite brown with part between both furrows dark brown, third tergite brown with medio-basal part dark brown, remaining segments of metasoma brown; ovipositor sheath dark brown, ovipositor red with apical part dark brown.

Distribution. China (Hainan)

Male. Unknown.

Diagnosis. The new species is similar to E. hoabinicus Belokobylskij \& Long, 2005 , but can be separated from the latter by having the first tergite more slender (also in lateral view) with more protruding spiracular tubercles, the grooves of the second tergite more convergent, second tergite irregularly reticulate, third tergite reticulate in medio-basal 0.55 and the colour of this area darker than the rest of third tergite.

Biological notes. Nothing is known about the hosts of this species.

Etymology. From "ret" (Latin meaning "net"), because of the irregularly reticulate second and third tergites of metasoma.

\section{Acknowledgement}

We thank Prof. Dr. C van Achterberg (Leiden, the Netherlands) for his critical review and comments of an earlier draft. We also thank two anonymous reviewers for their improvement of the text. This study was supported by the National Science Fund for Distinguished Young Scholars (No. 30625006), the National Scientific Foundation of China (Nos. 30499341, 30700063), the National S\&T Infrastructure Project (Nos. 2005DKA21402, 2005DKA21105), and the National Special Basic Research Funds (Nos. 2006FY110500-3, 2006FY120100).

\section{References}

Achterberg C van (1979) A revision of the subfamily Zelinae auct. (Hymenoptera, Braconidae). Tijdschrift voor Entomologie 122: 241- 479.

Achterberg C van (1988) Revision of the subfamily Blacinae Foerster (Hymenoptera: Braconidae). Zoologische Vergandelingen (Leiden) 249: 1-324.

Belokobylskij SA (1988) Three new species of braconid wasps of the subfamily Doryctinae (Hymenoptera, Braconidae) from Primorskii krai. Zoologicheskii Zhurnal 67(4): 625-629 (in Russian). 
Belokobylskij SA, Chen XX, Long KD (2005) Revision of the genus Eodendrus Belokobylskij (Hymenoptera: Braconidae, Doryctinae). Journal of Natural History 39 (29): 2715-2743. doi: $10.1080 / 00222930500114459$.

Granger C (1949) Braconides de Madagascar. Mémoires de l'Institut Scientifique de Madagas$\operatorname{car}(\mathrm{A}) 2: 1-428$.

Yu DS, Achterberg C van, Horstmann K (2005) World Ichneumonoidea 2004. Taxonomy, Biology, Morphology and Distribution. CD/DVD. Taxapad, Vancouver, Canada. 